Ueber die physiologische Verbrennung in den lebendigen Organismen. 641

\title{
Nachtrag zu meinem Aufsatz: \\ Ueber die physiologische Verbrennung in den lebendigen Organismen ${ }^{1}$ ).
}

Von

\section{E. Pfïger.}

Nach meiner Theorie der Lebensprocesse entsteht die Kohlensäure fortwährend im Innern sehr grosser Molecüle und versetzt durch die im Entstehungsmomente erfolgende Explosion alle Atome des zugehörigen Molecüles in mächtige Vibrationen. Mit andern Worten heisst dies: das aus nicht gasförmiger Materie plötzlich entstehende gasförmige Kohlensäuremolecill hat im Entstehungsmoment eine höhere Temperatur, die es seiner nächsten Umgebung mittheilt. Um zu beurtheilen, wie gross die Wirkung einer solchen Kohlensäureexplosion angeschlagen werden könne, erinnern wir uns, dass die lebendige Kraft der Atome des Molecüles der Temperatur proportional ist. Setzt man, um einen Anhalt zu haben, für einen Augenblick voraus, dass die ganze Verbrennungswärme des Kohlenstoffs in dem neugebildeten Kohlensäuremolecül anfänglich für einen Moment concentrirtsich befinde, so müsste seine Temperatur annähernd $10000^{\circ} \mathrm{C}$.

sein. Die Verbrennungswärme des Kohlenstoffs ist zu 8000 Wärmeeinheiten und die specifische Wärme der Kohlensäure zu 0,2 angesetzt.

Angesichts dieser riesigen Temperatur ist nun aber hervorzuheben, dass sicher unter allen Umständen schon während der Bildung der Kohlensäure Wärme durch Strahlung verloren geht.

Ebenso ist klar, dass bereits während der Bildung der Kohlensäure Kraft (und zwar z. B. chemische Spannkraft) in mechanische Arbeit (Hebung von Lasten) verwandelt werden kann, womit ich z. B. die Muskelcontraction erkläre. (Siehe dieses Archiv X, p. 329 und 344.)

In anderen Fällen können noch während des Bildungsacts der Kohlensäure z. B. beim Beginn ihrer Expansion Bestandtheile von

1) Siehe dies Archiv Bd. 10 p. 251. 
Molecülen (z. B. in den Drüsen) abgesprengt werden. Da hierbei eine starke Cohäsion überwunden wird, muss eine der geleisteten Arbeit entsprechende Wärme verschwinden.

Es ist für die durch die Oxydation erzeugte Temperatur des Augenblicks natürlich ferner nicht einerlei, ob die Kohlensäure aus $\mathrm{C}+\mathrm{O}+\mathrm{O}$ oder aus $\mathrm{CO}+\mathrm{O}$ entsteht. Aber doch hat eine Kohlenoxydflamme noch immer die enorme Temperatur von

$3042^{\circ} \mathrm{C}$.

Aus diesen und analogen Betrachtungen ergibt sich also mit Gewissheit, dass wir allerdings keine Berechtigung besitzen, das Vorkommen so hoher Temperaturen in der lebendigen Materie als sicher anzunehmen.

Wenn aber Physiologen und Chemiker von jeher sich darüber gewundert haben, dass im lebendigen Körper bei niederer Temperatur Oxydationsprocesse vorkommen, die, ausserhalb des Körpers im Allgemeinen nur bei hohen Temperaturen möglich sind, dann müssen wir jetzt sagen, dass die Voraussetzung einer niederen Temperatur, auf welche sich jenes Erstaunen stützte, von sehr zweifelhafter Sicherheit ist. Man hat nicht bedacht, dass die sogenannte niedere Körpertemperatur nur ein arithmetisches Mittel ist, welches unendlich viele höchst verschiedene Temperaturen unendlich vieler verschiedener Punkte eines Organes umfasst.

Die Explosion, oder die höhere Temperatur der an der Explosion betheiligten Atome hat aber noch aus dem Grund eine so grosse Bedeutung für das Leben, weil die Sprengpatrone in dem lebendigen Molecüle selbst sich entlädt. Mit anderen Worten: die höheren Temperaturen, die im Organismus auftreten können, müssen der Oertlichkeit halber, wo sie entstehen, gerade auf die lebendigen, d. h. beim Lebensprocess unmittelbar betheiligten Molecüle zunächst und vorzugsweise einwirken. Es ist vielleicht nicht überflüssig daran zu erinnern, dass nach meiner Theorie die lebendige Materie ein unendlich feines in Wasser aufgehangenes Fadennetz bildet, das also wegen seiner Feinheit und der ungeheuren Oberfläche sowie wegen der niederen Temperatur und der grossen Menge des Wassers sich unter den denkbar günstigsten Bedingungen zur Abkühlung befindet.

Was die Explosion unter günstigsten Verhältnissen in maximo annähernd zu leisten vermag, dafür bürgt die oben angegebene hohe Temperatur von $10000^{\circ}$. 
Sobald die Explosion in dem Molecüle eine Bresche gerissen, sind freie chemische Affinitäten in ihr vorhanden. Diese Bresche ist also wie ein gieriges beisskräftiges Maul, das mit Begierde zuschnappt. So findet Assimilation von Sauerstoff und brennbarem Material zur Sättigung der freigewordenen Affinitäten statt. Vielleicht ist auch von den Affinitäten der Bresche ein Molecül von Nahrungseiweiss gepackt worden. Dies wird nun auf das Heftigste geschüttelt; $d . h$. die energischen Vibrationen der lebendigen Molecüle pflanzen sich auf dasselbe fort; es wächst also seine Temperatur.

Die verstärkte lebendige Kraft, welche dem neu assimilirten Eiweissmolecüle zuertheilt worden ist, bedingt natürlich eine Vergrösserung der mittleren Abstände der Atome des Molecüles. Vergrösserung dieser Abstände bedentet aber Leistung von Arbeit.

Wie ich schon früher hervorhob, beschränke ich die Assimilation durchaus nicht nothwendig bloss auf die Bresche, weil die starke intramoleculare Bewegung alle Atome dem Status nascens nährt, wenn dies auch nur periodisch der Fall ist.

So ist mechanisch erklärt, wie die Assimilation geschieht und wie, auf Kosten der Wärme, das Gefüge des assimilirten Eiweissmolecüles gelockert worden ist.

Wenn die Atome eines chemischen Molecüles in heftige Schwingungen (durch Erhitzen, das gar nicht immer bis zu sehr hohen Temperaturen zu gehen braucht), versetzt werden, so wissen wir, dass Sauerstoff- und Wasserstoffatome dann gern sich als Wasser zusammenfinden, um auszutreten. Da nun aus Amiden durch Wasserverlust Cyanide entstehen, so kann man wegen der starken intramolecularen Bewegung der lebendigen Materie die Entstehung des Cyans im lebendigen Eiweiss wohl verstehen.

Die Bedeutung des Cyans für die Zersetzung im thierischen Organismus kann ich nunmehr einfach und ohne Hypothese erklären. Da alle Kraft des Lebens aus der Anziehung des Sauerstoffs zum Kohlenstoff und Wasserstoff stamint, lasse ich alle starke intramoleculare Bewegung in dem lebendigen Eiweissmolecül nur hierdurch, also besonders durch die Explosionen bedingt sein.

Da nun bekanntlich die nähere Beziehung, in welche der Kohlenstoff zum Stickstoff im Cyan tritt, unter verschiedenen Verhältnissen ungemein leicht wieder aufgehoben wird, indem z. B. der Kohlenstoff lieber mit Sauerstoff, der Stickstoff lieber mit Wasser- 
stoff sich verbindet, so reicht es für meine Erklärung aus, die leichte Zersetzbarkeit des Cyans als chemische Thatsache voranzustellen. Ohne dass ich hierbei dem Cyan eine besondere, hypothetische Eigenthümlichkeit, wie starke intramoleculare Bewegung der ponderabelen Atome zuschreibe, welche Annahme ich in meiner trüheren Abhandlung zu Hülfe genommen habe, liefert es relativ locker verknüpfte Fugen im Eiweissmolecüle überall da, wo Stickstoff und Kohlenstoff durch eine grössere Zahl von Affinitäten mit einander verbunden sind. Streng chemisch ist diese Zahl natürlich gleich drei.

So ist abgeleitet auf Grund der mechanischen Wärmetheorie die Verwandlung von Nahrungseiweiss in die andere Modification, welche ich lebendiges Eiweiss genannt habe.

So lange die Explosionen Breschen reissen, existiren Atome in statu nascenti; so lange findet Assimilation von Sauerstoff und nährenden Molecülen statt; so lange erzittert die lebendige Materie, und produzirt Wärme. Sobald aber die lebendigen Molecüle ihren intramolecularen kohlensäurebildenden Sauerstoff verbraucht haben, hören die Explosionen auf, also auch die Entstehung freier Affinitäten, also auch die Assimilationen. Das heisst mit andern Worten: das lebendige Molecül wird kalt, ruhig und indifferent, $d . h$. es stirbt. Nochmals werfe ich mir desshalb die Frage auf, die noch nicht gelöst ist: Kann die Wiederbelebung eines durch Sauerstoffentziehung "scheintodten " Organes erreicht werden, dessen letzter Rest an intramolecularem kohlensäurebildenden Sauerstoff absolut verbraucht ist? Ist also zur Wiederbelebung immer nothwendig, dass, wenigstens hier und da, im lebendigen Molecularnetz noch ein einziges Fünkchen glimmt oder doch eine noch auslösbare Patrone vorräthig geblieben?

Man versteht vielleicht auf diesem Weg, dass ein erstickter Mensch, obwohl sein Herz noch schlägt, durch beliebig lang fortgesetztes Lufteinblasen aus demselben Grund nicht wieder lebendig gemacht werden kann, aus dem kein noch so starker Luftstrom ein erloschenes Kohlenfeuer wieder entzündet.

Ich mache noch darauf aufmerksam, wie meine Hypothese die Continuität des Lebens erklärt.

Es versteht sich von selbst, dass für die Oxydation des Wasserstoffs theilweise analoge Betrachtungen wie für die des Kohlenstoffes angestellt werden können. Aber es ist ja nur circa 1/4-1/5 (Mittel) des eingeathmeten Sauerstoffs, der dem Wasserstoffe zu Gute kommt. 

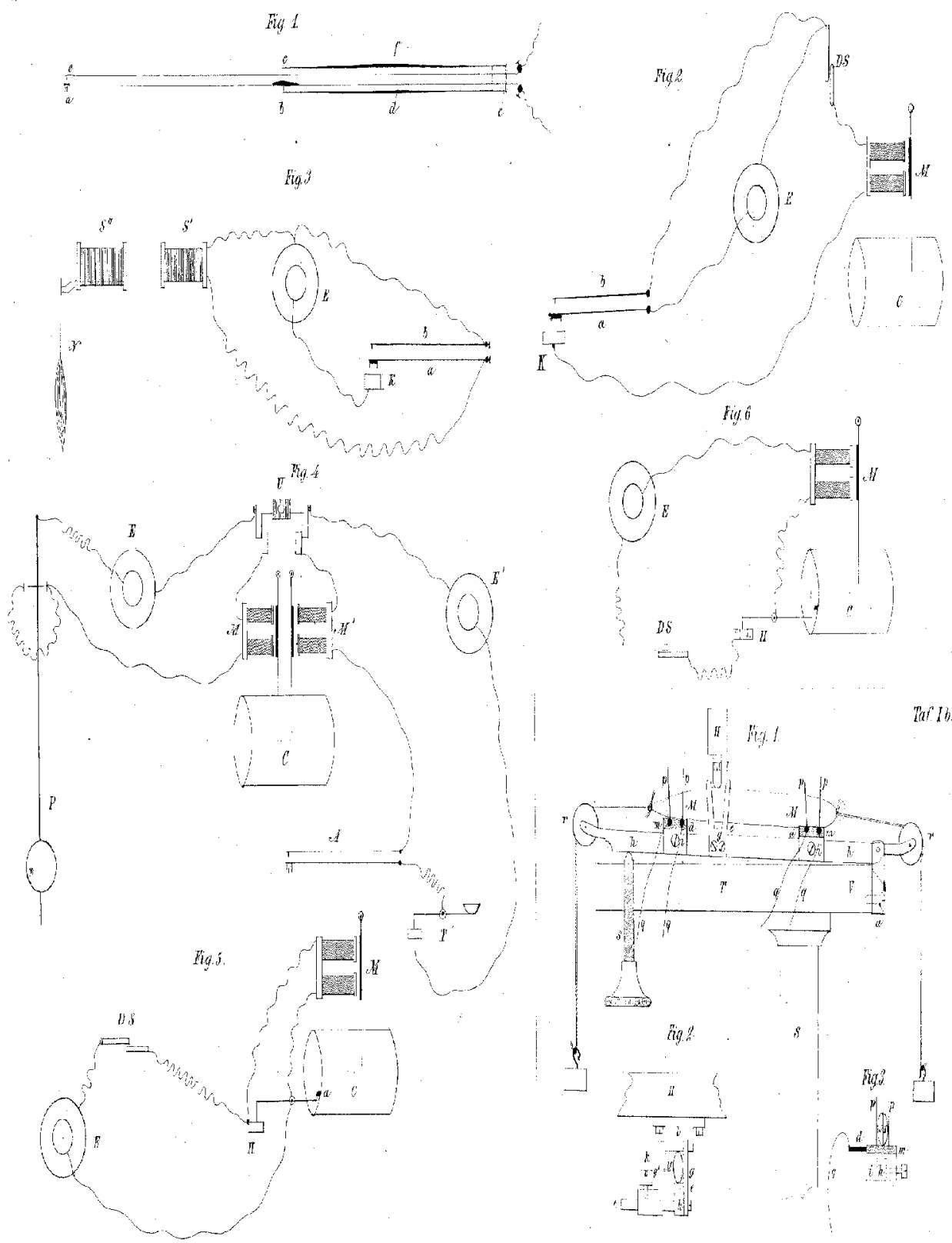